\title{
ON THE PICARD GROUP OF LOW-CODIMENSION SUBVARIETIES
}

\author{
ENRIQUE ARRONDO AND JORGE CARAVANTES
}

\begin{abstract}
We introduce a method to determine if $n$-dimensional smooth subvarieties of an ambient space of dimension at most $2 n-2$ inherit the Picard group from the ambient space (as it happens when the ambient space is a projective space, according to results of Barth and Larsen). As an application, we give an affirmative answer (up to some mild natural numerical conditions) when the ambient space is a Grassmannian of lines (thus improving results of Barth, Van de Ven and Sommese) or a product of two projective spaces of the same dimension.
\end{abstract}

A (complex) smooth subvariety of small codimension is expected to be very special. The most characteristic example of this principle is the case of subvarieties of the projective space, in which a famous conjecture of Hartshorne (see [7]) states that a smooth $n$-dimensional subvariety $X \subset \mathbb{P}^{N}$ must be a complete intersection if $2 N<3 n$. The main evidence for this conjecture is a series of results of Barth and Larsen showing that the topology of $X$ behaves like the one of a complete intersection. Specifically, it was first proved in $\left[3\right.$ that $H^{i}(X, \mathbb{Q}) \cong H^{i}\left(\mathbb{P}^{N}, \mathbb{Q}\right)$ if $i \leq 2 n-N$, then in [4] that $\pi_{1}(X)=0$ if $N \leq 2 n-1$ and finally in 9 that $H^{i}(X, \mathbb{Z}) \cong H^{i}\left(\mathbb{P}^{N}, \mathbb{Z}\right)$ if $i \leq 2 n-N$. In particular, this implies that the Picard group of $\operatorname{such} X$ is generated by the class of its hyperplane section if $N \leq 2 n-2$.

More generally, one can replace the projective space with other ambient spaces to study this kind of problems. For instance, in [5], Barth and Van de Ven prove that if $X$ is a smooth codimension-two subvariety of $\mathbb{G}(k, N)$ (the Grassmann variety of subspaces of dimension $k$ in $\left.\mathbb{P}^{N}\right)$, then $H^{i}(X, \mathbb{Z}) \cong H^{i}(G(k, N), \mathbb{Z})$ if $N-k \geq 6$ and $i=0,1,2$. A stronger result has been obtained by Sommese in 10, in the general framework of homogeneous varieties (with particularly strong results for Abelian varieties and products of Grassmannians). When particularized to $X \subset Y:=\prod \mathbb{G}\left(k_{l}, N_{l}\right)$ of dimension $n$, his result states that $\pi_{i}(Y, X)=0$ if $i \leq 2 n-2 \sum\left[\left(k_{l}+1\right)\left(N_{-} k_{l}\right)\right]+\min \left\{N_{l}\right\}+1$. As a consequence, if $Y=\mathbb{G}(k, N)$, then the Picard group of $X$ is generated by the hyperplane section when $2(k+1)(N-k)-N \leq 2 n-2$ (for $k=0$ this is the mentioned result for the projective space). We can also apply this to products of projective spaces and we get that, if $Y:=\mathbb{P}^{N} \times \mathbb{P}^{N}$, then the Picard group of $X$ is generated by the restrictions of the generators of $\operatorname{Pic}\left(\mathbb{P}^{N} \times \mathbb{P}^{N}\right)$ when $3 N+2 \leq 2 n$.

In this paper, we introduce a general method to study when the Picard group of an ambient space $Y$ of dimension $N$ is inherited by smooth subvarieties of dimension at least $\frac{N+2}{2}$ (hence in the range of Barth-Larsen result for projective spaces). Our starting point is the paper by the first author [1], where it is shown that the canonical divisor of any smooth fourfold in $\mathbb{G}(1,4)$ (and in $Q_{6}$ under some numerical conditions) is -numerically- a multiple of the hyperplane section. The method there relied strongly on the fact that the codimension was two (in order to use the so-called Hartshorne-Serre correspondence) and it seemed essential to work with the canonical divisor and not any other; a trivial application of Porteous formula produced a mysterious numerical relation, that eventually implied (via the Hodge index theorem) that the surface obtained by successive hyperplane sections was (numerically) subcanonical.

Both authors were partially supported by the MCYT project BFM2003-03971. 
Since Sommese's result indicates that the result of [1] should be true in higher codimension, we tried to understand where that mysterious numerical relation actually came from, without using such sophisticated techniques. We have thus found out that, in the general framework of $X \subset Y$ of dimension $n \geq \frac{N+2}{2}$, that relation could be easily obtained by just applying the selfintersection formula for $X$ in $Y$, the self-intersection formula for any smooth divisor $D$ of $X$ (this shows why it is important that $D$ has dimension at least its codimension in this ambient space) and the relation among them. In this way, we obtain in several ambient spaces (not necessarily with Picard number one) that any divisor (with only few restrictions) of $X$ must be numerically dependent on the generators of the Picard group of $Y$. When $X$ is also simply connected, this method shows that the Picard group of $X$ (which is then torsion-free) is generated (with rational coefficients) by the generators of the Picard group of $Y$.

Our first example, to which we devote Section 11 is $Y=\mathbb{P}^{N}$ and in this case we reobtain (up to divisibility) the theorem of Barth-Larsen for the Picard groups of subvarieties $X \subset \mathbb{P}^{N}$. In fact, as it happened in [1], the above mentioned numerical equality becomes $H_{\mid S}^{2} D_{\mid S}^{2}=\left(H_{\mid S} D_{\mid S}\right)^{2}$, where $S$ is the surface obtained intersecting $X$ with $n-2$ general hyperplanes. The Hodge index theorem implies immediately that $D_{\mid S}$ is numerically a multiple of $H_{\mid S}$. The Lefschetz hyperplane section and the simple connectedness of $X$ obtained in 4 completes the proof.

Following the idea of the projective case, in the second section we apply this method when the ambient space is a Grassmannian of lines (observe that our hypothesis about the relation between the dimension and codimension of $X$ are much milder than the ones in the result of Sommese). Here we prove that, when $X$ has positive intersection with all Schubert cycles of codimension $n$, then its Picard group has rank one. In this case, the key point is to write the numerical formula as a positive linear combination of Hodge-type expressions for the surfaces obtained by intersecting $X$ with the different Schubert cycles of codimension $n-2$ (which, by Hodge index theorem, must be positive, and they are zero when numerical equivalence occurs), plus some other positive terms.

In the third section we illustrate the fact that our method keep working even if the Picard group of the ambient space $Y$ has rank bigger than one. As a sample, we have chosen the case in which $Y$ is the cartesian product of two projective spaces of the same dimension. This particular choice has the advantage that $Y$ has even dimension (this is also one of the reasons why we choose $k=1$ in the case of Grassmannians), which simplifies the calculations. Again we prove in this case that, in the Barth-Larsen range, the Picard group of $X \subset Y$ is generated by two elements, assuming again some nonvanishing of the coefficients of the class of $X$ in the Chow ring of $Y$. This is again an improvement of the range given in [10, where the result is however stronger, in the sense that the Picard group of $X$ is proved to be generated by the restriction of the Picard group of the ambient space.

As a final section, we discuss the general validity of the method. We first briefly treat some other examples, in order to show that the method works also for non-homogeneous ambient spaces, and that in some cases extra assumptions are needed. We conclude with some general remarks and conjectures.

\section{Subvarieties of Projective SpaCe AND General Method}

In this section we will prove (up to divisibility of the hyperplane divisor class) Barth-Larsen theorem for the Picard group of subvarieties of projective spaces. This will illustrate the method that we will use later for different ambient varieties.

1.1. The Barth-Larsen result. First of all, we prove that the group of divisors up to numerical equivalence of a low codimension variety in $\mathbb{P}^{N}$ has rank 1. 
Proposition 1.1. Let $X$ be a nonsingular projective variety of dimension $n$ in $\mathbb{P}^{N}$ with $N \leq$ $2 n-2$ and let $H_{\mid X}$ denote the restriction to $X$ of the hyperplane class of $\mathbb{P}^{N}$. Then, for any divisor $D$ of $X$, there exists $q \in \mathbb{Q}$ so that $D \equiv_{\text {num }} q H_{\mid X}$.

Proof. Regarding $\mathbb{P}^{N}$ as a linear subspace of $\mathbb{P}^{2 n-2}$, we can assume $N=2 n-2$. Moreover, changing $D$ with $D+k H$ for a sufficient large $k \in \mathbb{Z}$, we can also assume that $D$ is very ample and hence smooth. Thus, we have the following exact sequence of vector bundles on $D$ :

$$
0 \rightarrow \mathcal{N}_{D / X} \rightarrow \mathcal{N}_{D / \mathbb{P}^{2 n-2}} \rightarrow\left(\mathcal{N}_{X / \mathbb{P}^{2 n-2}}\right)_{\mid D} \rightarrow 0
$$

Hence we get an equality for the top Chern classes:

$$
c_{n-1}\left(\mathcal{N}_{D / \mathbb{P}^{2 n-2}}\right)=c_{1}\left(\mathcal{N}_{D / X}\right) c_{n-2}\left(\left(\mathcal{N}_{\left.X / \mathbb{P}^{2 n-2}\right)}\right)_{\mid D}\right)
$$

Let $d$ and $\delta$ be the degrees of $X$ and $D$ in $\mathbb{P}^{2 n-2}$ respectively. In terms of the intersection of cycles in $X$ we have $d=H_{\mid X}^{n}$ and $\delta=D H_{\mid X}^{n-1}$. On the other hand, the self-intersection formula applied to each of the inclusions $D \subset X \subset \mathbb{P}^{2 n-2}$ yields

$$
\begin{aligned}
c_{n-1}\left(\mathcal{N}_{D / \mathbb{P}^{N}}\right)=\delta^{2} & =\left(D H_{\mid X}^{n-1}\right)^{2} \\
c_{1}\left(\mathcal{N}_{D / X}\right) & =D_{\mid D} \\
c_{n-2}\left(\mathcal{N}_{X / \mathbb{P}^{N}}\right) & =d H_{\mid D}^{n-2}
\end{aligned}
$$

Therefore (1.1) becomes:

$$
\left(D H_{\mid X}^{n-1}\right)^{2}=d D^{2} H_{\mid X}^{n-2}=H_{\mid X}^{n}\left(D^{2} H_{\mid X}^{n-2}\right)
$$

where all the intersection products are considered in $X$.

Taking $S$ to be the smooth irreducible surface obtained as intersection of $X$ with $n-2$ general hyperplanes, formula (1.2) becomes

$$
\left(D_{\mid S} H_{\mid S}\right)^{2}=D_{\mid S}^{2} H_{\mid S}^{2}
$$

Thus, Hodge index theorem implies that there exists $q \in \mathbb{Q}$ such that $D_{\mid S} \equiv_{\text {num }} q H_{\mid S}$. Finally, by Lefschetz's hyperplane theorem, the restriction map $H^{2}(X, \mathbb{Q}) \rightarrow H^{2}(S, \mathbb{Q})$ is injective, hence $D \equiv_{\text {num }} q H_{\mid X}$.

We obtain immediately from this the following consequence of the results of Barth and Larsen (observe that we have to use only their result about simple connectedness, which is in some sense weaker):

Corollary 1.2. In the hypothesis of Proposition 1.1 , PicX $\simeq \mathbb{Z}$.

Proof. Proposition 1.1 shows that Pic $X$ has rank one, so it is enough to show that it is torsion-free. But it is an immediate consequence of the fact that, as proved in [4], $X$ is simply connected. 
1.2. The general method. The above proofs suggest the following strategy to study when the Picard group is inherited (up to divisibility) by subvarieties of small codimension. Our general set-up will be a smooth ambient space $Y$ of dimension $N \geq 6$ and a smooth subvariety $X \subset Y$ of dimension $n$, and we will assume $N \leq 2 n-2$. For simplicity, assume that $N$ is even, so that after intersecting with $\frac{2 n-2-N}{2}$ general very ample divisors (and using Lefschetz's hyperplane theorem) we can assume $N=2 n-2$. The first step now is to take a smooth divisor $D$ and use the exact sequence

to obtain an equality

$$
0 \rightarrow \mathcal{N}_{D / X} \rightarrow \mathcal{N}_{D / Y} \rightarrow\left(\mathcal{N}_{X / Y}\right)_{\mid D} \rightarrow 0
$$

$$
c_{n-1}\left(\mathcal{N}_{D / Y}\right)-c_{1}\left(\mathcal{N}_{D / X}\right) c_{n-2}\left(\left(\mathcal{N}_{X / Y}\right)_{\mid D}\right)=0
$$

This equality becomes, using the self-intersection formulas of the three inclusions $D \subset X \subset Y$ the equality becomes

$$
P:=D \cdot D-D^{2} \cdot X_{\mid X}=0,
$$

where the first summand is a product in $Y$ and the second one is a product in $X$ (in this way, when $D=K$, this is exactly the "mysterious" equality (5) of [1] for a subvariety of $\mathbb{G}(1,4)$ ). The key ingredients to prove that the divisors on $X$ come (up to divisibility) from divisors on $Y$ will be then the following, corresponding respectively to Proposition 1.1] and Corollary 1.2 in the projective case:

Step (i): Derive from (1.4) and the Hodge index formula on the surface $S$ (the intersection of $n-2$ general very ample divisor on $X$ ) that the restriction of $D$ to the surface $S$ is numerically equivalent to a linear combination of restrictions of a set of generators of $\operatorname{Pic} Y$. This would imply, by Lefschetz's hyperplane theorem, that any divisor on $X$ is numerically equivalent (with rational coefficients) to the restriction of some divisor on $Y$.

Step (ii): If $X$ is simply connected, then from (i) we get that in fact any divisor has a multiple that is a restriction of a divisor on $Y$.

Observe that step (i) was immediate in the case of the projective space. In fact, equality (1.4) is equivalent to say (via the Hodge index theorem) that the restriction of $D$ to $S$ is a multiple of the hyperplane section. In general, this will be not so easy, and we will need to decompose $P$ as a sum of positive summands, as it happened in [1] when $Y=\mathbb{G}(1,4)$. Following this example, one should expect some of the summands to be Hodge formulas for the intersection of $X$ with suitable intersections of $X$ with cycles in $Y$ of codimension $n-2$ (one extra problem here is to show that these surfaces to which apply the Hodge index theorem are smooth irreducible). This is in fact the trickiest part of the method, and we do not see a way to decide a priori whether such a decomposition is possible or not.

About step (ii), as far as we know there are no general results that lead to expect that smooth subvarieties of small codimension are simply connected. We thus decided to concentrate in this paper on cases for which we now a priori that the simple connectedness hold. This is one of the reasons for having chosen Grassmannians and products of projective spaces, since Debarre proved in [6] the kind of result we needed (also the one about the irreducibility problem mentioned in the above paragraph).

\section{Subvarieties of Grassmannians of Lines}

In this section we apply the method we explained in the previous section to low-codimension subvarieties in Grassmannians. We will choose Grassmannians of lines because of two reasons. The first one is that, as we observed, it is convenient to work on an ambient space of even dimension. The second and main reason is that we need to use Schubert calculus, and the use of 
a compact notation can be very difficult and confusing. For instance, already for Grassmannians of lines in $\mathbb{P}^{n}$ the rank of the Chow ring in a given codimension depends on the parity of $n$. We thus decided to use integral parts in our expressions, hoping that this will not make the reading obscure; a reasonable choice could be to read this section twice: one of them thinking of $n=2 k$ and another one thinking of $n=2 k+1$. For Grassmannians of higher dimensional subspaces it seems better to study in each case the concrete Grassmannian that one could need to deal with.

2.1. Statement of the result and first remarks. Since we will need it continuously, we first recall the following:

Definition A Schubert cycle $\Omega(a, b)$ (with $0 \leq a<b \leq n$ ) is the class, in the Chow ring of $\mathbb{G}(1, n)$, of the $(a+b-1)$-dimensional Schubert variety $\Omega(A, B)$ consisting of the lines of $\mathbb{P}^{n}$ meeting the linear subspace $A$ and contained in the linear subspace $B$, where $A \subset B \subset \mathbb{P}^{n}$, $\operatorname{dim} A=a$ and $\operatorname{dim} B=b$. We refer to [8] for the needed background of Schubert cycles and their intersections.

The main result that we will prove is the following:

Theorem 2.1. Let $X \subset \mathbb{G}(1, n)$ be a smooth subvariety of dimension $n^{\prime} \geq n$ and let $H$ be denote the hyperplane class of $X$ (after the Plücker embedding). If all intersections of $X \cdot H^{n^{\prime}-n}$ with the Schubert cycles of codimension $n$ are different from zero, then PicX $\simeq \mathbb{Z}$.

Remark 2.2. Let $X \subset \mathbb{G}(1, n)$ be a smooth irreducible subvariety of codimension two. It will have class

$$
[X]=a_{1} \Omega(n-3, n)+a_{2} \Omega(n-2, n-1) .
$$

It is a simple exercise in Schubert calculus to see that

$$
[X] H=a_{1} \Omega(n-4, n)+\left(a_{1}+a_{2}\right) \Omega(n-3, n-1) .
$$

Hence, if $a_{1} \neq 0, X$ is in the hypothesis of Theorem 2.1 if $n \geq 5$, so that it is not necessary to impose $a_{2} \neq 0$. This shows that in general, when $n^{\prime}>n$, it is not necessary to impose the sufficient condition that the intersection of $X$ with all the Schubert cycles of codimension $n^{\prime}$ are different from zero.

Moreover, in these conditions, if $a_{1}=0$ this means that the union in $\mathbb{P}^{n}$ of the $(2 n-4)$ dimensional family of lines parametrized by $X$ has dimension at most $n-1$. This easily implies that $X$ is the Schubert variety of lines contained in a hyperplane of $\mathbb{P}^{n}$ and thus its Picard group is generated by its hyperplane section (and hence even in this case the thesis of the theorem remains true).

Also in the case $n=4$, having in mind that the intersection of $X$ with the Schubert cycles of lines in a general hyperplane of $\mathbb{P}^{4}$ is smooth, it is not difficult to check that $a_{1}, a_{2} \neq 0$ unless $X$ is the Schubert variety of lines in a general hyperplane of $\mathbb{P}^{4}$.

Summing up, Theorem 2.1 implies that the Picard group of any smooth subvariety $X \subset$ $\mathbb{G}(1, n)$ of codimension two is generated by one element if $n \geq 4$. This improves Barth, van de Ven and Sommese results in [5] and [10] (which require respectively $n \geq 7$ and $n \geq 6$ ), although unfortunately for $n=4,5$ we are not able to prove that we can take the hyperplane section as a generator of the Picard group.

Remark 2.3. In general, the numerical hypothesis in Theorem 2.1 are necessary. Consider for example the set of all the lines of a smooth quadric in $\mathbb{P}^{5}$. This is a smooth subvariety of $\mathbb{G}(1,5)$ of codimension 3 whose intersection with the Schubert cycle of all the lines passing through a point of $\mathbb{P}^{5}$ is clearly zero. On the other hand, identifying the smooth quadric with the Plücker embedding of $\mathbb{G}(1,3)$ in $\mathbb{P}^{5}$, it follows that $X$ is canonically isomorphic to the incidence variety in $\mathbb{P}^{3} \times \mathbb{P}^{3^{*}}$, whose Picard group has rank two. 
We now make some reductions to prove Theorem 2.1 in the line of subsection 1.2

Remark 2.4. Debarre has proved in [6. Corollaire 7.4] that an $X$ in the hypothesis of Theorem 2.1 is simply connected. Hence, as explained in subsection 1.2 we can assume $n^{\prime}=n$ and if $D$ is a smooth divisor of $X$ and $S$ is the intersection of $X$ with $n-2$ general hyperplanes, it is enough to prove $D_{\mid S} \equiv_{\text {num }} q H_{\mid S}$ for some $q \in \mathbb{Q}$.

We will devote the rest of the section to prove Theorem 2.1 according to Remark 2.4

2.2. General set-up. Now we state the notation for the numerical data that we will use throughout the section. We will fix a smooth $n$-dimensional subvariety $X \subset \mathbb{G}(1, n)$ with a smooth divisor $D$ on it. We will write the classes of $X$ and $D$ in the Chow ring of $\mathbb{G}(1, n)$ as

$$
[X]=a_{1} \Omega(1, n)+a_{2} \Omega(2, n-1)+\ldots+a_{\left[\frac{n}{2}\right]} \Omega\left(\left[\frac{n}{2}\right],\left[\frac{n+3}{2}\right]\right)
$$

(observe that the hypothesis about $X$ in the statement of Theorem 2.1] is saying that all $a_{i}$ are different from zero)

$$
[D]=\alpha_{1} \Omega(0, n)+\alpha_{2} \Omega(1, n-1)+\ldots+\alpha_{\left[\frac{n+1}{2}\right]} \Omega\left(\left[\frac{n-1}{2}\right],\left[\frac{n+2}{2}\right]\right) .
$$

A standard Schubert calculus provides then the following intersection table of cycles of $X$, which also defines the numbers $\lambda_{i}$ :

\begin{tabular}{c|cccccccc} 
& $\Omega(0, n)_{X}$ & $\Omega(1, n-1)_{X}$ & $\ldots$ & $\Omega\left(\left[\frac{n-1}{2}\right],\left[\frac{n+2}{2}\right]\right)_{X}$ & $D \Omega(1, n)_{X}$ & $D \Omega(2, n-1)_{X}$ & $\ldots$ & $D \Omega\left(\left[\frac{n}{2}\right],\left[\frac{n+3}{2}\right]\right)_{X}$ \\
\hline$H_{X}$ & $a_{1}$ & $a_{1}+a_{2}$ & $\ldots$ & $a_{\left[\frac{n-1}{2}\right]}+a_{\left[\frac{n+1}{2}\right]}$ & $\alpha_{1}+\alpha_{2}$ & $\alpha_{2}+\alpha_{3}$ & $\ldots$ & $\alpha_{\left[\frac{n}{2}\right]}+\alpha_{\left[\frac{n+2}{2}\right]}$ \\
$D$ & $\alpha_{1}$ & $\alpha_{2}$ & $\ldots$ & $\alpha_{\left[\frac{n+1}{2}\right]}$ & $\lambda_{1}$ & $\lambda_{2}$ & $\ldots$ & $\lambda_{\left[\frac{n}{2}\right]}$
\end{tabular}

(where the notation $\Omega(i, j)_{X}$ represents the restriction to $X$ of the Schubert cycle $\Omega(i, j)$ ).

Remark 2.5. Observe that, if $n$ is odd, the term $a_{\left[\frac{n+1}{2}\right]}$ is not defined, so that we will take it to be zero. In the same way, we define $\alpha_{\left[\frac{n+2}{2}\right]}$ to be zero if $n$ is even. To have a compact expression for the matrix we can also set $a_{0}=0$. As we remarked at the beginning of the section, this compact expression, although convenient for avoiding unnecessary repetitions, can be confusing. Hence for the convenience of the reader we write the precise form of the above matrix of intersections depending on whether $n$ is even or odd:

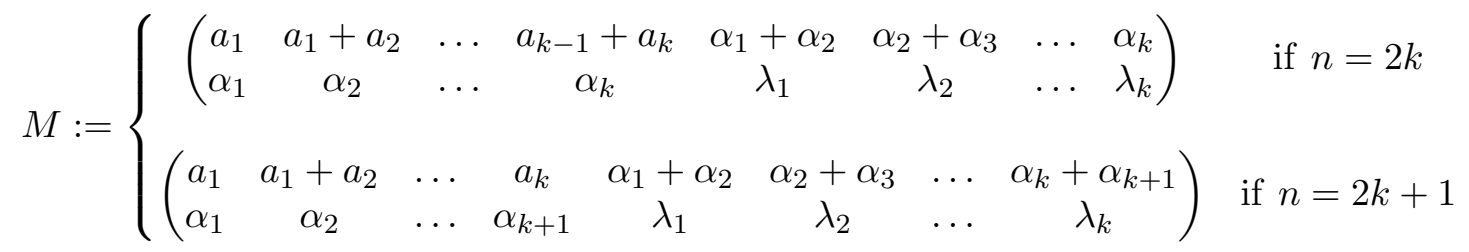

If $D$ is numerically a multiple of $H_{X}$, it is clear that the matrix $M$ must have rank one. Reciprocally, we claim that if $M$ has rank one, then Theorem 2.1 follows. Indeed if there is $q \in \mathbb{Q}$ such that the second row of $M$ is $q$ times the first one, this means that the product of

$$
D^{\prime}:=D-q H_{X}
$$

with any $\Omega(i, n-i)_{X}$ and any $D \Omega(j, n+1-j)_{X}$ is zero. Since $H_{X}^{n-1}$ and $H_{X}^{n-2}$ are respectively a linear combination of Schubert cycles of the type $\Omega(i, n-i)_{X}$ and $\Omega(j, n+1-j)_{X}$, it follows that $D^{\prime} H_{X}^{n-1}=D^{\prime} D H_{X}^{n-2}=0$. Hence, if $S$ is the intersection of $X$ with $n-2$ general hyperplanes, it follows:

$$
D_{\mid S}^{\prime} H_{\mid S}=D^{\prime} H_{X}^{n-1}=0
$$




$$
D_{\mid S}^{\prime 2}=D^{\prime}\left(D-q H_{X}\right) H_{X}^{n-2}=0
$$

By Hodge index theorem, we conclude that $D_{\mid S}^{\prime}$ is numerically equivalent to zero. By Remark 2.4. Theorem 2.1 follows.

We will thus devote the rest of the section to prove that $M$ has rank one (Corollary 2.9).

\subsection{End of the proof.}

Lemma 2.6. The following equality holds.

$$
P:=\alpha_{1}^{2}+\ldots+\alpha_{\left[\frac{n+1}{2}\right]}^{2}-a_{1} \lambda_{1}-\ldots-a_{\left[\frac{n}{2}\right]} \lambda_{\left[\frac{n}{2}\right]}=0
$$

Proof. This is (1.4) using (2.1), (2.2) and the intersection products given by Schubert calculus and the matrix $M$.

Remark 2.7. For all $i=1, \ldots,\left[\frac{n}{2}\right]$, let $S_{i}$ be the surface obtained intersecting $X$ with a general $\Omega(i, n-i+1)$. It is easy to see that $S_{i}$ is nonsingular, and by [6. Théorème 8.1] it is also irreducible.

Applying Hodge index theorem to $S_{i}$ and using the fact that $H \Omega(i, n-i+1)=\Omega(i-1, n-$ $i+1)+\Omega(i, n-i)$, we get

$$
0 \geq \text { Hodge }_{i}:=\left|\begin{array}{cc}
H_{\mid S_{i}}^{2} & D_{\mid S_{i}} H_{\mid S_{i}} \\
D_{\mid S_{i}} H_{\mid S_{i}} & D_{\mid S_{i}}^{2}
\end{array}\right|=\left|\begin{array}{cc}
a_{i-1}+a_{i} & \alpha_{i}+\alpha_{i+1} \\
\alpha_{i} & \lambda_{i}
\end{array}\right|+\left|\begin{array}{cc}
a_{i}+a_{i+1} & \alpha_{i}+\alpha_{i+1} \\
\alpha_{i+1} & \lambda_{i}
\end{array}\right|
$$

(as usual, one should be careful with the correct expression of $\operatorname{Hodge}_{i}$ in the cases $i=1$, [ $\left[\frac{n}{2}\right]$; in particular, notice that, if $n$ is even and $i=\left[\frac{n}{2}\right]$, then the correct definition of Hodge $_{i}$ consists only of the first summand of (2.5) ).

Lemma 2.8. With $P$ and Hodge $_{i}$ defined by (2.4) and (2.5), the following equality holds:

$$
P=-\sum_{i=1}^{\left[\frac{n}{2}\right]} \frac{a_{i}}{a_{i-1}+2 a_{i}+a_{i+1}} \operatorname{Hodge}_{i}+\sum_{i=1}^{\left[\frac{n+1}{2}\right]-1} \frac{a_{i}\left|\begin{array}{cc}
a_{i-1}+a_{i} & a_{i}+a_{i+1} \\
\alpha_{i} & \alpha_{i+1}
\end{array}\right|^{2}}{\left(a_{i-1}+2 a_{i}+a_{i+1}\right)\left(a_{i-1}+a_{i}\right)\left(a_{i}+a_{i+1}\right)}
$$

Proof. We regard the expressions as polynomials in the indeterminates $\alpha_{1}, \ldots, \alpha_{\left[\frac{n+1}{2}\right]}, \lambda_{1}, \ldots$, $\lambda_{\left[\frac{n}{2}\right]}$ with coefficients in the quotient field of $\mathbb{C}\left[a_{1}, \ldots, a_{k}\right]$. The only non-zero coefficients are those of the monomials of the type $\alpha_{i}^{2}, \alpha_{i} \alpha_{i+1}, \lambda_{i}$. Hence it suffices to check that the corresponding coefficients in the left and right hand sides coincide. This is trivial for the coefficient of $\lambda_{i}$, which is $a_{i}$ in both sides.

For $\alpha_{i} \alpha_{i+1}$, its coefficient is zero in the left-hand side of (2.6), while in the right-hand side it is:

$$
2 \frac{a_{i}}{a_{i-1}+2 a_{i}+a_{i+1}}-2 \frac{a_{i}}{\left(a_{i-1}+2 a_{i}+a_{i+1}\right)\left(a_{i-1}+a_{i}\right)\left(a_{i}+a_{i+1}\right)}\left(a_{i}+a_{i+1}\right)\left(a_{i-1}+a_{i}\right)
$$

which is also zero.

For $\alpha_{i}^{2}$, its coefficient in the left-hand side is 1 , while in the right-hand side is:

$$
\begin{array}{r}
\frac{a_{i-1}}{a_{i-2}+2 a_{i-1}+a_{i}}+\frac{a_{i}}{a_{i-1}+2 a_{i}+a_{i+1}}+\frac{a_{i-1}\left(a_{i-2}+a_{i-1}\right)^{2}}{\left(a_{i-2}+2 a_{i-1}+a_{i}\right)\left(a_{i-2}+a_{i-1}\right)\left(a_{i-1}+a_{i}\right)} \\
+\frac{a_{i}\left(a_{i}+a_{i+1}\right)^{2}}{\left(a_{i-1}+2 a_{i}+a_{i+1}\right)\left(a_{i-1}+a_{i}\right)\left(a_{i}+a_{i+1}\right)}
\end{array}
$$


and a simple calculation shows that this is also 1 .

We leave to the reader to check that all these equalities remain valid when $i$ takes the minimum and maximum values; a special care should be taken when $n$ is even, because (as observed at the end of Remark 2.7), the expression $a_{i-1}+2 a_{i}+a_{i+1}$ appearing in both sums of (2.6) must be regarded as $\left(a_{i-1}+a_{i}\right)+\left(a_{i}+a_{i+1}\right)$, thus just $a_{i-1}+a_{i}$ if $i=\frac{n}{2}$.

Corollary 2.9. The matrix $M$ of (2.3) has rank one.

Proof. The left-hand side of (2.6) is zero by Lemma 2.6 while the right-hand side is a sum of nonnegative terms by (2.5). Therefore we get equalities

$$
\begin{gathered}
\left|\begin{array}{cc}
a_{i-1}+a_{i} & \alpha_{i}+\alpha_{i+1} \\
\alpha_{i} & \lambda_{i}
\end{array}\right|+\left|\begin{array}{cc}
a_{i}+a_{i+1} & \alpha_{i}+\alpha_{i+1} \\
\alpha_{i+1} & \lambda_{i}
\end{array}\right|=0 \\
\left|\begin{array}{cc}
a_{i-1}+a_{i} & a_{i}+a_{i+1} \\
\alpha_{i} & \alpha_{i+1}
\end{array}\right|=0
\end{gathered}
$$

(observe that for this it was crucial the hypothesis $a_{i} \neq 0$ of Theorem 2.1). The first equalities imply that the columns of $M$ containing a $\lambda_{i}$ depend on the other columns, while the second inequalities show that the columns not containing any $\lambda_{i}$ span a one-dimensional subspace.

\section{Subvarieties of Products of PRojeCtive SPACES}

In this section we will apply our method to a product of two projective spaces of the same dimension. The main result we will prove is the following:

Theorem 3.1. Let $X \subset \mathbb{P}^{n-1} \times \mathbb{P}^{n-1}$ be smooth of dimension greater than or equal to $n$ such that the two projection maps $X \rightarrow \mathbb{P}^{n-1}$ are surjective. Then PicX is a free abelian group of rank two, in which the pullbacks $\mathrm{H}_{1}$ and $\mathrm{H}_{2}$ of the hyperplane classes of each $\mathbb{P}^{n-1}$ are linearly independent.

Remark 3.2. It is clear that the hypothesis of the surjectivity of the projections $X \rightarrow \mathbb{P}^{n-1}$ are necessary. For instance, if the image of the first projection is contained in a subvariety $Z \subset \mathbb{P}^{n-1}$ with Picard group of rank at least two, then we can find many smooth subvarieties $X \subset Z \times \mathbb{P}^{n-1}$ whose Picard group has rank at least three (for example taking successive hyperplane sections of the Segre embedding of $\mathbb{P}^{n-1} \times \mathbb{P}^{n-1}$ ). To give a concrete example, just consider the embedding $\mathbb{P}^{1} \times \mathbb{P}^{1} \times \mathbb{P}^{1} \times \mathbb{P}^{1} \subset \mathbb{P}^{3} \times \mathbb{P}^{3}$, (in which we identify the first and last $\mathbb{P}^{1} \times \mathbb{P}^{1}$ with a smooth quadric in $\mathbb{P}^{3}$ after the Segre embedding).

On the other hand, without any condition about surjectivity, the results of [10] show that $\operatorname{Pic} X$ is generated by $H_{1}$ and $H_{2}$ if $\operatorname{dim} X \geq \frac{3 n-1}{2}$.

Remark 3.3. After the result of Debarre showing that $X$ is simply connected 6, Corollaire 2.4], as remarked in subsection [1.2 it is enough to assume $\operatorname{dim} X=n$ and prove that every smooth divisor $D$ on $X$ restricted to the intersection of $X$ with $n-2$ general hyperplanes (i.e. divisors in the class $H_{1}+H_{2}$ ) is numerically equivalent to a rational combination of $H_{1}$ and $H_{2}$. Observe that $H_{1}, H_{2}$ are linearly independent, since $H_{1}^{n}=H_{2}^{n}=0$ and $\left(H_{1}+H_{2}\right)^{n}>0$. 
3.1. General set-up. We fix now the general notation that we will use throughout the section. Let $X$ be a smooth $n$-dimensional subvariety of $\mathbb{P}^{n-1} \times \mathbb{P}^{n-1}$ such that the two projections $X \rightarrow \mathbb{P}^{n-1}$ are surjective. Let $D$ be a smooth divisor of $X$ and let $H_{1}, H_{2}$ be the pullback to $X$ of the hyperplane classes of $\mathbb{P}^{n-1}$. We define $a_{i}, \alpha_{i}$, and $\lambda_{i}$ as the intersection products in $X$ according to the following table (sometimes it will be practical to set $a_{0}=a_{n}=0$ in order to get compact expressions):

\begin{tabular}{c|cccccccccc} 
& $H_{1}^{n-1}$ & $H_{1}^{n-2} H_{2}$ & $H_{1}^{n-3} H_{2}^{2}$ & $\ldots$ & $H_{2}^{n-1}$ & $D H_{1}^{n-2}$ & $D H_{1}^{n-3} H_{2}$ & $D H_{1}^{n-4} H_{2}^{2}$ & $\ldots$ & $D H_{2}^{n-2}$ \\
\hline$H_{1}$ & 0 & $a_{1}$ & $a_{2}$ & $\ldots$ & $a_{n-1}$ & $\alpha_{1}$ & $\alpha_{2}$ & $\alpha_{3}$ & $\ldots$ & $\alpha_{n-1}$ \\
$H_{2}$ & $a_{1}$ & $a_{2}$ & $a_{3}$ & $\ldots$ & 0 & $\alpha_{2}$ & $\alpha_{3}$ & $\alpha_{4}$ & $\ldots$ & $\alpha_{n}$ \\
$D$ & $\alpha_{1}$ & $\alpha_{2}$ & $\alpha_{3}$ & $\ldots$ & $\alpha_{n}$ & $\lambda_{1}$ & $\lambda_{2}$ & $\lambda_{3}$ & $\ldots$ & $\lambda_{n-1}$
\end{tabular}

If $D$ depends numerically on $H_{1}$ and $H_{2}$, it is clear that the intersection matrix

$$
M:=\left(\begin{array}{cccccccccc}
0 & a_{1} & a_{2} & \ldots & a_{n-1} & \alpha_{1} & \alpha_{2} & \alpha_{3} & \ldots & \alpha_{n-1} \\
a_{1} & a_{2} & a_{3} & \ldots & 0 & \alpha_{2} & \alpha_{3} & \alpha_{4} & \ldots & \alpha_{n} \\
\alpha_{1} & \alpha_{2} & \alpha_{3} & \ldots & \alpha_{n} & \lambda_{1} & \lambda_{2} & \lambda_{3} & \ldots & \lambda_{n-1}
\end{array}\right)
$$

must have rank at least two. We claim that in fact if $M$ has rank two then Theorem 3.1 follows. Indeed, observe first that $\left|\begin{array}{cc}0 & a_{n-1} \\ a_{1} & 0\end{array}\right| \neq 0$, since this is exactly the hypothesis that the projections $X \rightarrow \mathbb{P}^{n-1}$ are surjective. Hence, if $M$ has rank two, it follows that the third row is a linear combination of the first two. This implies that there are $p, q \in \mathbb{Q}$ such that the product of

$$
D^{\prime}:=D-p H_{1}-q H_{2}
$$

with $H_{1}^{n-1}, H_{1}^{n-2} H_{1}, \ldots, H_{2}^{n-1}, D H_{1}^{n-2}, D H_{1}^{n-3} H_{2}, \ldots, D H_{2}^{n-2}$ is zero. If $S$ is the intersection of $X$ with $n-2$ general hyperplanes, then it follows that

$$
\begin{gathered}
D_{\mid S}^{\prime}\left(H_{1}+H_{2}\right)_{\mid S}=D^{\prime}\left(H_{1}+H_{2}\right)^{n-1}=0 \\
D_{\mid S}^{\prime 2}=D^{\prime}\left(D-p H_{1}-q H_{2}\right)\left(H_{1}+H_{2}\right)^{n-2}=0 .
\end{gathered}
$$

Therefore, by Hodge index theorem $D_{\mid S}^{\prime}$ is numerically equivalent to zero. By Remark 3.3. this implies Theorem 3.1

We will thus devote the rest of the section to prove that $M$ has rank two (Corollary [3.16).

3.2. First numerical properties. In this subsection we prove the first relations between the numerical invariants of a smooth subvariety $X \subset \mathbb{P}^{n-1} \times \mathbb{P}^{n-1}$ of dimension $n$.

Observe that the intersection matrix (3.1) shows that the classes of $X$ and $D$ in the Chow ring of $\mathbb{P}^{n-1} \times \mathbb{P}^{n-1}$ are

$$
\begin{gathered}
{[X]=a_{n-1} H_{1}^{n-2}+a_{n-2} H_{1}^{n-3} H_{2}+\ldots+a_{1} H_{2}^{n-2}} \\
{[D]=\alpha_{n} H_{1}^{n-1}+\alpha_{n-1} H_{1}^{n-2} H_{2}+\ldots+\alpha_{1} H_{2}^{n-1}}
\end{gathered}
$$

(we abuse momentarily the notation by indicating with $H_{1}, H_{2}$ the hyperplane classes in each $\mathbb{P}^{n-1}$, instead of denoting their pullback to $\left.X\right)$. This implies the following:

Lemma 3.4. The following equality holds

$$
P:=\alpha_{1} \alpha_{n}+\alpha_{2} \alpha_{n-1}+\ldots+\alpha_{n} \alpha_{1}-a_{1} \lambda_{n-1}-a_{2} \lambda_{n-2}-\ldots-a_{n-1} \lambda_{1}=0
$$


Proof. This is (1.4) using (3.2), (3.3) and the intersection products given by the matrix $M$ of (3.1).

A first important observation about our numerical invariants is that, in our situation, all the numbers $a_{1}, \ldots, a_{n-1}$ are different from zero:

Lemma 3.5. Let $X \subset \mathbb{P}^{n-1} \times \mathbb{P}^{n-1}$ be irreducible of dimension $n$. Let $a_{1}, \ldots, a_{n-1}$ be defined as above. Suppose that $a_{1}, a_{n-1}$ are different from zero. Then all $a_{i}$ are strictly positive.

Proof. See [6, Théorème 1.3].

Definition For $i=1, \ldots, n-1$, we will denote $S_{i}$ to the surface obtained intersecting $X$ with a general $\mathbb{P}^{i} \times \mathbb{P}^{n-i}$ (hence the class of $S_{i}$ in $X$ is $H_{1}^{n-i-1} H_{2}^{i-1}$ ). Since both natural projections restricted to $X$ are surjective, it follows from [6. Théorème 1.3] that $S_{i}$ is a smooth irreducible surface.

Remark 3.6. The Hodge index theorem applied to the divisors $H_{1}, H_{2}$ restricted to $S_{i}$ implies the inequality:

$$
\left|\begin{array}{cc}
a_{i-1} & a_{i} \\
a_{i} & a_{i+1}
\end{array}\right|=\left|\begin{array}{cc}
H_{1 \mid S_{i}}^{2} & H_{1 \mid S_{i}} H_{2 \mid S_{i}} \\
H_{1 \mid S_{i}} H_{2 \mid S_{i}} & H_{2 \mid S_{i}}^{2}
\end{array}\right| \leq 0
$$

with equality if and only if $H_{1}$ and $H_{2}$ are numerically dependent on $S_{i}$. Lemma 3.5 allows us to write all these inequalities as:

$$
\frac{a_{1}}{a_{2}} \leq \frac{a_{2}}{a_{3}} \leq \ldots \leq \frac{a_{n-2}}{a_{n-1}} .
$$

Thus it follows that, for all $i<j$, we have

$$
\left|\begin{array}{cc}
a_{i-1} & a_{j} \\
a_{i} & a_{j+1}
\end{array}\right| \leq 0
$$

with equality if and only if

i.e.

$$
\left|\begin{array}{cc}
a_{i-1} & a_{i} \\
a_{i} & a_{i+1}
\end{array}\right|=\left|\begin{array}{cc}
a_{i} & a_{i+1} \\
a_{i+1} & a_{i+2}
\end{array}\right|=\ldots=\left|\begin{array}{cc}
a_{j-1} & a_{j} \\
a_{j} & a_{j+1}
\end{array}\right|=0
$$

$$
\frac{a_{i-1}}{a_{i}}=\frac{a_{i}}{a_{i+1}}=\ldots=\frac{a_{j}}{a_{j+1}} .
$$

We suspect that the inequalities in (3.5) are never equalities. However we are not able to prove it, so that our proofs will become sometimes more complicated when considering the cases in which some equalities hold.

Remark 3.7. We apply now the Hodge index theorem to the restriction to $S_{i}$ of the divisors $H_{1}, H_{2}, D$ and get

$$
r_{i, i+1, n+i}:=\left|\begin{array}{ccc}
a_{i-1} & a_{i} & \alpha_{i} \\
a_{i} & a_{i+1} & \alpha_{i+1} \\
\alpha_{i} & \alpha_{i+1} & \lambda_{i}
\end{array}\right|=\left|\begin{array}{ccc}
H_{1 \mid S_{i}}^{2} & H_{1 \mid S_{i}} H_{2 \mid S_{i}} & H_{1 \mid S_{i}} D_{\mid S_{i}} \\
H_{1 \mid S_{i}} H_{2 \mid S_{i}} & H_{2 \mid S_{i}}^{2} & H_{2 \mid S_{i}} D_{\mid S_{i}} \\
H_{1 \mid S_{i}} D_{\mid S_{i}} & H_{2 \mid S_{i}} D_{\mid S_{i}} & D_{\mid S_{i}}^{2}
\end{array}\right| \geq 0
$$

(in general $r_{i, j, k}$ will denote the minor of the columns $i, j, k$ of the matrix $M$ ) with equality if and only if $H_{1}, H_{2}$ and $D$ are numerically dependent when restricted to $S_{i}$. Hence the vanishing of $r_{i, i+1, n+i}$ would immediately prove that the restriction of $D$ to $S_{i}$ is numerically a combination of $H_{1}$ and $H_{2}$, unless the restrictions to $S_{i}$ of $H_{1}$ and $H_{2}$ are numerically dependent. 
In this latter case (which occurs if and only if $\frac{a_{i-1}}{a_{i}}=\frac{a_{i}}{a_{i+1}}$, by Remark 3.6) the restriction of $D$ to $S_{i}$ is numerically a combination of $H_{1}$ and $H_{2}$ if and only if it is just a multiple of $H_{1}$. By the Hodge index theorem, this is equivalent to the inequality:

$$
\tilde{r}_{i}:=\left|\begin{array}{cc}
a_{i-1} & \alpha_{i} \\
\alpha_{i} & \lambda_{i}
\end{array}\right|=\left|\begin{array}{cc}
H_{1 \mid S_{i}}^{2} & H_{1 \mid S_{i}} D_{\mid S_{i}} \\
H_{1 \mid S_{i}} D_{\mid S_{i}} & D_{\mid S_{i}}^{2}
\end{array}\right| \leq 0
$$

to be an equality. Moreover, it easily follows that

$$
\operatorname{rk}\left(\begin{array}{ccc}
a_{i-1} & a_{i} & \alpha_{i} \\
a_{i} & a_{i+1} & \alpha_{i+1}
\end{array}\right)=1
$$

since this is the multiplication matrix in $S_{i}$ of the divisors $H_{1}, H_{2}$ (which are numerically dependent) with $H_{1}, H_{2}, D$. In particular

$$
\alpha_{i+1}=\frac{a_{i+1}}{a_{i}} \alpha_{i}
$$

Remark 3.8. The above remark shows that we should check how many equalities we have in the chain (3.5). A practical way to control this is to define the function:

$$
\begin{array}{ccc}
\sigma:\{2, \ldots, n\} & \longrightarrow & \{2, \ldots, n\} \\
i & \mapsto \quad \frac{a_{l-2}}{a_{l-1}}<\frac{a_{l-1}}{a_{l}}=\ldots=\frac{a_{i-2}}{a_{i-1}}=\frac{a_{i-1}}{a_{i}}
\end{array}
$$

In this way, the equality $\frac{a_{i-1}}{a_{i}}=\frac{a_{j-1}}{a_{j}}$ is equivalent to the equality $\sigma(i)=\sigma(j)$. In other words, the equalities in (3.5) coincide with the equalities in

$$
\sigma(2) \leq \sigma(3) \leq \ldots \leq \sigma(n) .
$$

Observe that saying that there are no equalities in (3.5) (as we said, this is what we suspect) is equivalent to saying that $\sigma$ is the identity map.

We recall from Remark 3.6 that $\frac{a_{\sigma(i)-2}}{a_{\sigma(i)-1}}<\frac{a_{\sigma(i)-1}}{a_{\sigma(i)}}=\ldots=\frac{a_{i-2}}{a_{i-1}}=\frac{a_{i-1}}{a_{i}}$ is equivalent to $\left|\begin{array}{cc}a_{\sigma(i)-1} & a_{i-1} \\ a_{\sigma(i)} & a_{i}\end{array}\right|=0, \quad\left|\begin{array}{cc}a_{\sigma(i)-2} & a_{i-1} \\ a_{\sigma(i)-1} & a_{i}\end{array}\right|<0$. On the other hand, iterating (3.8) we get

$$
\alpha_{i}=\frac{a_{i}}{a_{\sigma(i)}} \alpha_{\sigma(i)}
$$

The idea of the above function $\sigma$ is that it allows to determine "virtual" equalities in the chain (3.5), and then perform the substitutions given in (3.10). This will be very useful in the next section, when $a_{1}, \ldots, a_{n-1}$ will be indeterminates rather than concrete values. This motivates the following definition, which indicates when a function $\sigma$ is obtained as in the above remark:

Definition We call partition map to a function $\sigma:\{2, \ldots, n\} \rightarrow\{2, \ldots, n\}$ satisfying:

(1) $\sigma(i) \leq i$ for all $i$

(2) for all $j \in\{\sigma(i), \ldots, i\}, \sigma(j)=\sigma(i)$.

3.3. Polynomial equalities. Throughout this subsection, we will consider $a_{i}, \alpha_{i}, \lambda_{i}$ as indeterminates, and we will consider the polynomial ring $\mathbb{Q}\left(a_{1}, \ldots, a_{n-1}\right)\left[\alpha_{1}, \ldots, \alpha_{n}, \lambda_{1}, \ldots, \lambda_{n-1}\right]$. In this context, the elements of $\mathbb{Q}\left(a_{1}, \ldots, a_{n-1}\right)$ will be regarded as coefficients (our convention $a_{0}=a_{n}=0$ will be useful again for writing the expressions in a compact way). We define the polynomials (compare with (3.4), (3.6) and (3.7)):

$$
P:=\alpha_{1} \alpha_{n}+\alpha_{2} \alpha_{n-1}+\ldots+\alpha_{n} \alpha_{1}-a_{1} \lambda_{n-1}-a_{2} \lambda_{n-2}-\ldots-a_{n-1} \lambda_{1}
$$


and the coefficients

$$
\begin{aligned}
r_{i, i+1, n+i} & :=\left|\begin{array}{ccc}
a_{i-1} & a_{i} & \alpha_{i} \\
a_{i} & a_{i+1} & \alpha_{i+1} \\
\alpha_{i} & \alpha_{i+1} & \lambda_{i}
\end{array}\right| \\
\tilde{r}_{i} & :=\left|\begin{array}{ccc}
a_{i-1} & \alpha_{i} \\
\alpha_{i} & \lambda_{i}
\end{array}\right| \\
r_{i, i+1, n-i+1} & :=\left|\begin{array}{ccc}
a_{i-1} & a_{i} & a_{n-i} \\
a_{i} & a_{i+1} & a_{n-i+1} \\
\alpha_{i} & \alpha_{i+1} & \alpha_{n-i+1}
\end{array}\right|
\end{aligned}
$$

$$
\begin{gathered}
c_{i}:=\frac{a_{n-i}}{\left|\begin{array}{cc}
a_{i-1} & a_{i} \\
a_{i} & a_{i+1}
\end{array}\right|} \\
d_{i}:=\frac{a_{i} a_{n-i}}{\left|\begin{array}{cc}
a_{i-1} & a_{i} \\
a_{i} & a_{i+1}
\end{array}\right|\left|\begin{array}{cc}
a_{i-1} & a_{n-i} \\
a_{i} & a_{n-i+1}
\end{array}\right|\left|\begin{array}{cc}
a_{i} & a_{n-i} \\
a_{i+1} & a_{n-i+1}
\end{array}\right|}
\end{gathered}
$$

Observe that, if $i=\left\lceil\frac{n}{2}\right\rceil$ then $r_{i, i+1, n-i+1}=0$ and $d_{i}$ is undefined. We will take both of them equal to zero.

Lemma 3.9. The following equality holds in $\mathbb{Q}\left(a_{1}, \ldots, a_{n-1}\right)\left[\alpha_{1}, \ldots, \alpha_{n}, \lambda_{1}, \ldots, \lambda_{n-1}\right]$ :

$$
P=-\sum_{i=1}^{n-1} c_{i} r_{i, i+1, n+i}-\sum_{i=1}^{n-1} d_{i} r_{i, i+1, n-i+1}^{2}
$$

Proof. We have to compare the coefficients of the different monomials. The only monomials appearing in (3.13) are of the type $\lambda_{i}, \alpha_{i}^{2}, \alpha_{i} \alpha_{i+1}, \alpha_{i} \alpha_{n-i+1}$ and $\alpha_{i} \alpha_{n-i+2}$. It is immediate that the coefficient of $\lambda_{i}$ is $-a_{n-i}$ in both the left and right hand sides of (3.13).

For $\alpha_{i}^{2}$ (we do not consider the case $i=1,\left\lceil\frac{n}{2}\right\rceil,\left\lceil\frac{n}{2}\right\rceil+1, n$, which overlaps with other cases), we have zero on the left-hand side, while the coefficient on the right-hand side comes from $r_{i-1, i, n+i-1}, r_{i, i+1, n+i}, r_{i-1, i, n-i+2}^{2}, r_{n-i+1, n-i+2, i}^{2}$ and $r_{i, i+1, n-i+1}^{2}$ and it is

$$
c_{i-1} a_{i-2}+c_{i} a_{i+1}-d_{i-1}\left|\begin{array}{cc}
a_{i-2} & a_{n-i+1} \\
a_{i-1} & a_{n-i+2}
\end{array}\right|^{2}-d_{i}\left|\begin{array}{cc}
a_{i} & a_{n-i} \\
a_{i+1} & a_{n-i+1}
\end{array}\right|^{2}-d_{n-i+1}\left|\begin{array}{cc}
a_{n-i} & a_{n-i+1} \\
a_{n-i+1} & a_{n-i+2}
\end{array}\right|^{2}
$$

which is also zero.

For $\alpha_{i} \alpha_{i+1}$, (we assume now $i \neq\left\lceil\frac{n}{2}\right\rceil,\left\lceil\frac{n}{2}\right\rceil+1$ in order to avoid overlaps with other cases) we have again zero on the left-hand side, while the coefficient on the right-hand side comes from $r_{i, i+1, n+i}$ and $r_{i, i+1, n-i+1}^{2}$, and it is

$$
-2 c_{i} a_{i}+2 d_{i}\left|\begin{array}{cc}
a_{i-1} & a_{n-i} \\
a_{i} & a_{n-i+1}
\end{array}\right|\left|\begin{array}{cc}
a_{i} & a_{n-i} \\
a_{i+1} & a_{n-i+1}
\end{array}\right|
$$

which is obviously zero.

For $\alpha_{i} \alpha_{n-i+1}$ (assuming now $i \neq 1,\left\lceil\frac{n}{2}\right\rceil,\left\lceil\frac{n}{2}\right\rceil+1$ ), the coefficient on the left-hand side is 2 , while on the right-hand side, it comes from $r_{i, i+1, n-i+1}^{2}$ and $r_{n-i+1, n-i+2, i}^{2}$, and it is

$$
-2 d_{i}\left|\begin{array}{cc}
a_{i} & a_{n-i} \\
a_{i+1} & a_{n-i+1}
\end{array}\right|\left|\begin{array}{cc}
a_{i-1} & a_{i} \\
a_{i} & a_{i+1}
\end{array}\right|+2 d_{n-i+1}\left|\begin{array}{cc}
a_{n-i} & a_{n-i+1} \\
a_{n-i+1} & a_{n-i+2}
\end{array}\right|\left|\begin{array}{cc}
a_{i-1} & a_{n-i+1} \\
a_{i} & a_{n-i+2}
\end{array}\right|
$$

which is also 2 . 
For $\alpha_{i} \alpha_{n-i+2}$ (again with $i \neq\left\lceil\frac{n}{2}\right\rceil,\left\lceil\frac{n}{2}\right\rceil+1$ ), its coefficient is zero in the left-hand side, while it appears only in the right-hand side in $r_{i-1, i, n-i+2}^{2}$ and $r_{n-i+1, n-i+2, i}^{2}$, with coefficient:

$$
2 d_{i-1}\left|\begin{array}{cc}
a_{i-2} & a_{n-i+1} \\
a_{i-1} & a_{n-i+2}
\end{array}\right|\left|\begin{array}{cc}
a_{i-2} & a_{i-1} \\
a_{i-1} & a_{i}
\end{array}\right|+2 d_{n-i+1}\left|\begin{array}{cc}
a_{n-i} & a_{n-i+1} \\
a_{n-i+1} & a_{n-i+2}
\end{array}\right|\left|\begin{array}{cc}
a_{i-1} & a_{n-i} \\
a_{i} & a_{n-i+1}
\end{array}\right|
$$

which is zero.

We are left with the coefficients of the cases we have excluded, i.e. the ones of the monomials $\alpha_{1}^{2}, \alpha_{1} \alpha_{n}, \alpha_{k}^{2}, \alpha_{k} \alpha_{k+1}, \alpha_{k} \alpha_{k+2}, \alpha_{k+1}^{2}, \alpha_{k+1} \alpha_{k+2}$ and $\alpha_{n}^{2}$, with $k=\left\lceil\frac{n}{2}\right\rceil$. We omit the proof, which is as before a simple computation, but depending now on whether $n=2 k$ or $n=2 k-1$ for the cases involving $k$ in an index.

Thinking of our application to subvarieties of $\mathbb{P}^{n-1} \times \mathbb{P}^{n-1}$, Lemma 3.9 could be the analogue of Lemma 2.8 but a priori the denominators of some coefficient $c_{i}, d_{i}$ could vanish, depending on whether we have equalities in the chain (3.5). If this happens, the idea would be to use, when necessary, the inequalities (3.7) instead of (3.6) and perform the substitutions (3.10). In order to keep all these substitutions in the framework of polynomials, we fix a partition map $\sigma$ for the rest of the subsection.

The first case in which we would be in trouble is when the denominator of $c_{i}$ vanishes (i.e. when $\sigma(i)=\sigma(i+1)$, in terms of the partition map). As we observed in Remark 3.7 in this case we should replace $r_{i, i+1, n+i}$ with $\tilde{r}_{i}$ (with an appropriate coefficient to fit in (3.13)). On the other hand, the denominator of $d_{i}$ would also create problems, so it looks convenient to remove the term $d_{i} r_{i, i+1, n-i+1}^{2}$ from (3.13). The precise way of performing these changes is given by the following:

Lemma 3.10. Suppose $\sigma(i)=\sigma(i+1)$ for some $i=1, \ldots, n-1$ and set:

Then,

$$
\begin{array}{r}
l_{i}:=\frac{a_{n-i}\left|\begin{array}{ll}
a_{i} & a_{n-i+1} \\
\alpha_{i} & \alpha_{n-i+1}
\end{array}\right| \begin{array}{cc}
a_{i-1} & a_{i} \\
a_{i} & a_{i+1}
\end{array} \mid}{a_{i}\left|\begin{array}{cc}
a_{i-1} & a_{n-i} \\
a_{i} & a_{n-i+1}
\end{array}\right| \begin{array}{cc}
a_{i} & a_{n-i} \\
a_{i+1} & a_{n-i+1}
\end{array} \mid} \\
m_{i}:=\frac{a_{n-i}\left|\begin{array}{cc}
a_{i} & a_{i+1} \\
\alpha_{i} & \alpha_{i+1}
\end{array}\right|\left(a_{i} a_{n-i+1} \alpha_{i+1}-2 a_{i} a_{i+1} \alpha_{n-i+1}+a_{i+1} a_{n-i+1} \alpha_{i}\right)}{a_{i} a_{i+1}\left|\begin{array}{cc}
a_{i} & a_{n-i} \\
a_{i+1} & a_{n-i+1}
\end{array}\right|}
\end{array}
$$

As a consequence,

$$
-c_{i} r_{i, i+1, n+i}-d_{i} r_{i, i+1, n-i+1}^{2}=-\frac{a_{n-i}}{a_{i+1}} \tilde{r}_{i}-l_{i}-m_{i}
$$

$$
\begin{aligned}
P=- & \sum_{\sigma(i)<\sigma(i+1)} c_{i} r_{i, i+1, n+i} \\
& -\sum_{\sigma(i)=\sigma(i+1)} \frac{a_{n-i}}{a_{i+1}} \tilde{r}_{i}- \\
& -\sum_{\sigma(i)<\sigma(i+1)} d_{i} r_{i, i+1, n-i+1}^{2}-\sum_{\sigma(i)=\sigma(i+1)} l_{i}-\sum_{\sigma(i)=\sigma(i+1)} m_{i}
\end{aligned}
$$

Proof. The first part is a straightforward computation, while the second one follows immediately from the first one and (3.13).

Observe that we can still have problems with the denominator of $l_{i}$ and $m_{i}$ if $\sigma(n-i+1)$ equals $\sigma(i)=\sigma(i+1)$. In the same way, even if $\sigma(i)<\sigma(i+1)$ we can have problems with the 
denominator of $d_{i}$ if $\sigma(n-i+1)$ equals $\sigma(i)$ or $\sigma(i+1)$. Let us see that we can avoid these problems by performing the substitutions suggested by (3.10). We thus make the following:

Definition Given any polynomial $Q \in \mathbb{Q}\left(a_{1}, \ldots, a_{n-1}\right)\left[\alpha_{1}, \ldots, \alpha_{n}, \lambda_{1}, \ldots, \lambda_{n-1}\right]$, we define a new polynomial (depending on few variables):

$$
Q^{\sigma}:=Q\left(a_{1}, \ldots, a_{n-1}, \alpha_{1}, \frac{a_{2}}{a_{\sigma(2)}} \alpha_{\sigma(2)}, \ldots, \frac{a_{n-1}}{a_{\sigma(n-1)}} \alpha_{\sigma(n-1)}, \alpha_{n}, \lambda_{1}, \ldots, \lambda_{n-1}\right)
$$

We see now how these substitutions affect the problematic terms in (3.14).

Lemma 3.11. The following identities hold:

(i) If $\sigma(i)=\sigma(i+1)$, then $m_{i}^{\sigma}=0$.

(ii) If $\sigma(i)=\sigma(i+1)=\sigma(n-i+1)$, then $l_{i}^{\sigma}=0$.

(iii) If $\sigma(i)<\sigma(i+1)=\sigma(n-i+1)$, then $r_{i, i+1, n-i+1}^{\sigma}=\frac{-a_{i}}{a_{\sigma(i)} a_{i+1}}\left|\begin{array}{cc}a_{i} & a_{n-i} \\ a_{i+1} & a_{n-i+1}\end{array}\right|\left|\begin{array}{cc}a_{\sigma(i)} & a_{i+1} \\ \alpha_{\sigma(i)} & \alpha_{i+1}\end{array}\right|$ and hence

$$
g_{i}:=d_{i}\left(r_{i, i+1, n-i+1}^{\sigma}\right)^{2}=\frac{a_{i}^{3} a_{n-i}\left|\begin{array}{cc}
a_{i} & a_{n-i} \\
a_{i+1} & a_{n-i+1}
\end{array}\right|\left|\begin{array}{cc}
a_{\sigma(i)} & a_{i+1} \\
\alpha_{\sigma(i)} & \alpha_{i+1}
\end{array}\right|^{2}}{a_{\sigma(i)}^{2} a_{i+1}^{2}\left|\begin{array}{cc}
a_{i-1} & a_{n-i} \\
a_{i} & a_{n-i+1}
\end{array}\right|\left|\begin{array}{cc}
a_{i-1} & a_{i} \\
a_{i} & a_{i+1}
\end{array}\right|}
$$

(iv) If $\sigma(n-i+1)=\sigma(i)<\sigma(i+1)$, then $r_{i, i+1, n-i+1}^{\sigma}=\frac{-1}{a_{\sigma(i)}}\left|\begin{array}{cc}a_{i-1} & a_{n-i} \\ a_{i} & a_{n-i+1}\end{array}\right|\left|\begin{array}{cc}a_{\sigma(i)} & a_{i+1} \\ \alpha_{\sigma(i)} & \alpha_{i+1}\end{array}\right|$ and hence

$$
h_{i}:=d_{i}\left(r_{i, i+1, n-i+1}^{\sigma}\right)^{2}=\frac{a_{i} a_{n-i}\left|\begin{array}{cc}
a_{i-1} & a_{n-i} \\
a_{i} & a_{n-i+1}
\end{array}\right|\left|\begin{array}{cc}
a_{\sigma(i)} & a_{i+1} \\
\alpha_{\sigma(i)} & \alpha_{i+1}
\end{array}\right|^{2}}{a_{\sigma(i)}^{2}\left|\begin{array}{cc}
a_{i} & a_{n-i} \\
a_{i+1} & a_{n-i+1}
\end{array}\right|\left|\begin{array}{cc}
a_{i-1} & a_{i} \\
a_{i} & a_{i+1}
\end{array}\right|}
$$

Proof. This is just a straightforward computation.

Corollary 3.12. With the above notations,

$$
\begin{aligned}
& P^{\sigma}=-\sum_{\sigma(i)<\sigma(i+1)} c_{i} r_{i, i+1, n+i}^{\sigma}-\sum_{\sigma(i)=\sigma(i+1)} \frac{a_{n-i}}{a_{i+1}} \tilde{r}_{i}^{\sigma}- \\
& -\sum_{\sigma(n-i+1) \neq \sigma(i)<\sigma(i+1) \neq \sigma(n-i+1)} d_{i}\left(r_{i, i+1, n-i+1}^{\sigma}\right)^{2}-\sum_{\sigma(i)<\sigma(i+1)=\sigma(n-i+1)} g_{i}- \\
& -\sum_{\sigma(n-i+1)=\sigma(i)<\sigma(i+1)} h_{i}-\sum_{\sigma(i)=\sigma(i+1) \neq \sigma(n-i+1)} l_{i}^{\sigma}
\end{aligned}
$$

Proof. Just apply Lemma 3.11 to the equality (3.14) of Lemma 3.10.

3.4. End of the proof. After the previous technical subsection we come back to the geometrical situation of a subvariety $X \subset \mathbb{P}^{n-1} \times \mathbb{P}^{n-1}$ with the usual invariants. We can now state and prove the analogue of Lemma 2.8 
Lemma 3.13. The following equality holds:

$$
0=-\sum_{\frac{a_{i-1}}{a_{i}}<\frac{a_{i}}{a_{i+1}}} \frac{a_{n-i}}{\left|\begin{array}{cc}
a_{i-1} & a_{i} \\
a_{i} & a_{i+1}
\end{array}\right|}\left|\begin{array}{ccc}
a_{i-1} & a_{i} & \alpha_{i} \\
a_{i} & a_{i+1} & \alpha_{i+1} \\
\alpha_{i} & \alpha_{i+1} & \lambda_{i}
\end{array}\right|-\sum_{\frac{a_{i-1}}{a_{i}}=\frac{a_{i}}{a_{i+1}}} \frac{a_{n-i}}{a_{i+1}}\left|\begin{array}{cc}
a_{i+1} & \alpha_{i+1} \\
\alpha_{i+1} & \lambda_{i}
\end{array}\right|-
$$

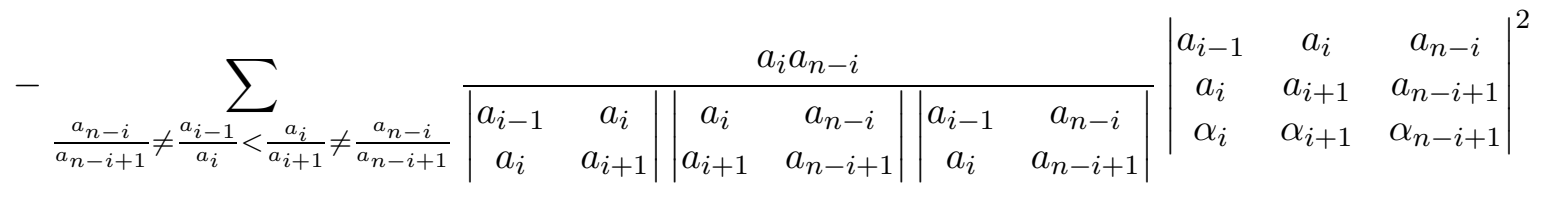

and therefore

$$
\begin{gathered}
\left|\begin{array}{ccc}
a_{i-1} & a_{i} & \alpha_{i} \\
a_{i} & a_{i+1} & \alpha_{i+1} \\
\alpha_{i} & \alpha_{i+1} & \lambda_{i}
\end{array}\right|=0 \quad \text { if } \frac{a_{i-1}}{a_{i}}<\frac{a_{i}}{a_{i+1}} \\
\left|\begin{array}{cc}
a_{i+1} & \alpha_{i+1} \\
\alpha_{i+1} & \lambda_{i}
\end{array}\right|=0 \quad \text { if } \frac{a_{i-1}}{a_{i}}=\frac{a_{i}}{a_{i+1}} \\
\left|\begin{array}{ccc}
a_{i-1} & a_{i} & a_{n-i} \\
a_{i} & a_{i+1} & a_{n-i+1} \\
\alpha_{i} & \alpha_{i+1} & \alpha_{n-i+1}
\end{array}\right|=0 \quad \text { if } \frac{a_{n-i}}{a_{n-i+1}} \neq \frac{a_{i-1}}{a_{i}}<\frac{a_{i}}{a_{i+1}} \neq \frac{a_{n-i}}{a_{n-i+1}}
\end{gathered}
$$

Proof. Let $\sigma$ be the partition map defined by (3.9). Then (3.18) is an easy consequence of the equality (3.17) in Corollary 3.12. Indeed, observe first that, by (3.10), any polynomial $Q \in \mathbb{Q}\left(a_{1}, \ldots, a_{n-1}\right)\left[\alpha_{1}, \ldots, \alpha_{n}, \lambda_{1}, \ldots, \lambda_{n-1}\right]$, takes the same value as $Q^{\sigma}$ when applied to the invariants of $X$, provided that all denominators are different from zero. In particular, $P^{\sigma}$ vanishes thanks to Lemma 3.4. We thus apply Corollary 3.12 and analyze each of the summands of (3.17):

It is clear that the first three summands of (3.17) coincide with the summands of (3.18) and that their denominators do not vanish (recall that this is equivalent to say $\sigma(i)<\sigma(i+1)$ ). For the other summands of (3.17), if $\frac{a_{i-1}}{a_{i}}<\frac{a_{i}}{a_{i+1}}=\frac{a_{n-i}}{a_{n-i+1}}$ or $\frac{a_{n-i}}{a_{n-i+1}}=\frac{a_{i-1}}{a_{i}}<\frac{a_{i}}{a_{i+1}}$, then $d_{i}\left(r_{i, i+1, n-i+1}^{\sigma}\right)^{2}$ takes the value $g_{i}$ or $h_{i}$ respectively of (3.15) and (3.16), whose denominators do not vanish, but their numerators contain respectively the factor $\left|\begin{array}{cc}a_{i-1} & a_{n-i} \\ a_{i} & a_{n-i+1}\end{array}\right|$ and $\left|\begin{array}{cc}a_{i} & a_{n-i} \\ a_{i+1} & a_{n-i+1}\end{array}\right|$, so that they vanish; and finally, if $\frac{a_{i-1}}{a_{i}}=\frac{a_{i}}{a_{i+1}} \neq \frac{a_{n-i}}{a_{n-i+1}}$, then the denominator of $l_{i}$, as defined in Lemma 3.10 does not vanish, while the numerator contains the factor $\left|\begin{array}{cc}a_{i-1} & a_{i} \\ a_{i} & a_{i+1}\end{array}\right|$, hence it vanishes.

Observe now that all the summands of (3.18) are nonnegative by Remark 3.7, which implies the equalities (3.19), (3.20) and (3.21) (again, it was crucial to know that all $a_{i}$ are not zero).

We are now ready to prove that the matrix $M$ of (3.1) has rank two. We will do it in several steps. 
Corollary 3.14. For each $i=1, \ldots, n, i \neq\left\lceil\frac{n}{2}\right\rceil$, define the following submatrix of $M$ :

$$
M_{i}^{\prime}:=\left\{\begin{array}{lll}
\left(\begin{array}{ccc}
a_{i-1} & a_{i} & a_{n-i} \\
a_{i} & a_{i+1} & a_{n-i+1} \\
\alpha_{i} & \alpha_{i+1} & \alpha_{n-i+1}
\end{array}\right) & \text { if } i<n-i \\
\left(\begin{array}{ccc}
a_{n-i} & a_{i-1} & a_{i} \\
a_{n-i+1} & a_{i} & a_{i+1} \\
\alpha_{n-i+1} & \alpha_{i} & \alpha_{i+1}
\end{array}\right) & \text { if } n-i<i-1
\end{array}\right.
$$

Then the middle column of $M_{i}^{\prime}$ is a linear combination of the two others.

Proof. This is immediate if $\frac{a_{n-i}}{a_{n-i+1}} \neq \frac{a_{i-1}}{a_{i}}<\frac{a_{i}}{a_{i+1}} \neq \frac{a_{n-i}}{a_{n-i+1}}$, since in this case any two columns of $M_{i}^{\prime}$ are linearly independent, and (3.21) implies that any of the columns of $M_{i}^{\prime}$ is a linear combination of the two others. It is also immediate if $\frac{a_{i-1}}{a_{i}}=\frac{a_{i}}{a_{i+1}}$, since in this case $\left(\begin{array}{c}a_{i-1} \\ a_{i} \\ \alpha_{i}\end{array}\right)$ and $\left(\begin{array}{c}a_{i} \\ a_{i+1} \\ \alpha_{i+1}\end{array}\right)$ are multiple of each other (by the second part of Remark [3.7) and one of them is the middle column. We are thus left with the cases $\frac{a_{i-1}}{a_{i}}<\frac{a_{i}}{a_{i+1}}=\frac{a_{n-i}}{a_{n-i+1}}$ and $\frac{a_{n-i}}{a_{n-i+1}}=\frac{a_{i-1}}{a_{i}}<\frac{a_{i}}{a_{i+1}}$, which are proved in the same way using again Remark 3.7.

Corollary 3.15. The submatrix

$$
M^{\prime}:=\left(\begin{array}{cccccc}
0 & a_{1} & a_{2} & \ldots & a_{n-2} & a_{n-1} \\
a_{1} & a_{2} & a_{3} & \ldots & a_{n-1} & 0 \\
\alpha_{1} & \alpha_{2} & \alpha_{3} & \ldots & \alpha_{n-1} & \alpha_{n}
\end{array}\right)
$$

of $M$ has rank two.

Proof. It is enough to prove for each $i=1, \ldots, n$ that the $i$-th column of $M^{\prime}$ is a linear combination of the first and last columns (which in turn are linearly independent). We will prove it by induction on $d(i):=\min \{i-1, n-i\}$ (i.e. the distance of $i$ to the border of the interval $[1, n])$. Of course, the statement is trivial for $d(i)=0$ (i.e. $i=1$ or $i=n$ ).

Assume first $i<\frac{n+2}{2}$ (hence $d(i)=i-1$ ). Then Corollary 3.14 implies that the $i$-th column is a linear combination of the $(i-1)$-th and $(n-i+2)$-th columns. By induction hypothesis (observe that $d(i-1)=d(n-i+2)=i-2)$, these two columns are in turn linear combinations of the first and last columns of $M$, which completes the proof of this case.

If instead $i>\frac{n+1}{2}$ (hence $d(i)=n-i$ ), Corollary 3.14 implies now that the $i$-th column is a linear combination of the $(n-i+1)$-th and $(i+1)$-th columns. The $(i+1)$-th column is, again by induction hypothesis, a linear combination of the first and last columns of $M$. For the $(n-i+1)$-th column we observe that $d(n-i+1)=n-i=d(i)$ and that $n-i+1<\frac{n+2}{2}$. Hence we are in the situation of the previous case, for which we already proved the inductive step.

Corollary 3.16. The matrix $M$ of (3.1) has rank two.

Proof. By Corollary 3.15, it is enough to prove that any column of $M$ containing a $\lambda_{i}$ is a linear combination of the columns of $M^{\prime}$. To this purpose, for $i=1, \ldots, n-1$ we consider the 
submatrix:

$$
M_{i}:=\left(\begin{array}{ccc}
a_{i-1} & a_{i} & \alpha_{i} \\
a_{i} & a_{i+1} & \alpha_{i+1} \\
\alpha_{i} & \alpha_{i+1} & \lambda_{i}
\end{array}\right)
$$

If $\frac{a_{i-1}}{a_{i}}<\frac{a_{i}}{a_{i+1}}$, then the two first columns of $M_{i}$ are linearly independent, and (3.19) proves that the last column of $M_{i}$ is a linear combination of the first two, as wanted.

If instead $\frac{a_{i-1}}{a_{i}}=\frac{a_{i}}{a_{i+1}}$, then by (3.8)

$$
\left|\begin{array}{cc}
a_{i} & \alpha_{i} \\
a_{i+1} & \alpha_{i+1}
\end{array}\right|=0
$$

which, together with (3.20) and $a_{i+1} \neq 0$, implies that the last column of $M_{i}$ is a multiple of the middle one.

\section{Further EXAMPles AND REMARKS}

We start with several examples that illustrate until which point the method explained in subsection 1.2 can work. In two of them, we will not have any result stating that subvarieties in the chosen ambient space $Y$ are simply connected, so that step (ii) of the method cannot be applied. Anyway, the key point in the method is step (i), in order to prove that the divisors on subvarieties of $X$ come numerically from divisors on $Y$. We will not do all the details, which can be found in the forthcoming $\mathrm{PhD}$ thesis of the second author. We start with a negative example.

Example 4.1. Let $X \subset Q_{2 n-2}$ be a smooth $n$-dimensional subvariety of the smooth $(2 n-2)$ dimensional quadric. It is well known that the class of $X$ in the Chow ring of $Q_{2 n-2}$ takes the form $d H^{n-2}$, where $H$ is the hyperplane class. On the other hand, if $D$ is a (smooth) divisor on $X$, its class as a cycle in $Q_{2 n-2}$ will be $[D]=\alpha_{1} A_{1}+\alpha_{2} A_{2}$, where $A_{1}, A_{2}$ are the classes of the two families of $(n-1)$-dimensional linear spaces contained in $Q_{2 n-2}$. Having in mind the intersection of two linear spaces in $Q_{2 n-2}$ depending on the parity of $n$, equality (1.4) becomes

$$
P:=\left\{\begin{array}{cc}
2 \alpha_{1} \alpha_{2}-d D^{2} H^{n-2}=0 & \text { if } n \text { is even } \\
\alpha_{1}^{2}+\alpha_{2}^{2}-d D^{2} H^{n-2}=0 & \text { if } n \text { is odd }
\end{array}\right.
$$

If $S$ is the intersection of $X$ with $n-2$ general hyperplanes we can thus write (observe that $\left.D_{S} H_{S}=D H^{n-1}=\alpha_{1}+\alpha_{2}\right)$

$$
P= \begin{cases}\frac{1}{2}\left(\left(D_{S} H_{S}\right)^{2}-D_{S}^{2} H_{S}^{2}\right)-\frac{1}{2}\left(\alpha_{1}-\alpha_{2}\right)^{2} & \text { if } n \text { is even } \\ \frac{1}{2}\left(\left(D_{S} H_{S}\right)^{2}-D_{S}^{2} H_{S}^{2}\right)+\frac{1}{2}\left(\alpha_{1}-\alpha_{2}\right)^{2} & \text { if } n \text { is odd. }\end{cases}
$$

This proves (using the Hodge index theorem for $S$ ) that, when $n$ is odd, any divisor on $X$ is numerically a multiple of the hyperplane section; moreover, since $X$ is a smooth subvariety of $\mathbb{P}^{2 n-1}$, it follows from [4] that $X$ is simply connected, and hence we conclude that its Picard group is isomorphic to $\mathbb{Z}$. However, when $n$ is even, we get that $D$ is a multiple of $H$ only when $\alpha_{1}=\alpha_{2}$, i.e. $D A_{1}=D A_{2}$. Since the equality $D A_{1}=D A_{2}$ defines one linear condition on PicX (which could be identically zero), it follows that PicX has rank at most two (we need to use again that $X$ is simply connected). The rank-two case can actually occur: consider the Segre 
embedding $\mathbb{P}^{1} \times \mathbb{P}^{n-1} \hookrightarrow \mathbb{P}^{2 n-1}$, whose image can be defined as the subvariety $X$ consisting of those point for which the matrix

$$
\left(\begin{array}{cccc}
X_{0} & X_{1} & \ldots & X_{n-1} \\
X_{n} & X_{n+1} & \ldots & X_{2 n-1}
\end{array}\right)
$$

has rank one. Since $n$ is even, it follows that $X$ is contained in the smooth quadric $Q_{2 n-2}$ of equation

$$
\left|\begin{array}{cc}
X_{0} & X_{1} \\
X_{n} & X_{n+1}
\end{array}\right|+\left|\begin{array}{cc}
X_{2} & X_{3} \\
X_{n+2} & X_{n+3}
\end{array}\right|+\ldots+\left|\begin{array}{cc}
X_{n-2} & X_{n-1} \\
X_{2 n-2} & X_{2 n-1}
\end{array}\right|=0
$$

Strengthening Conjecture 4.5 of [1], we suspect that the above example is the only smooth $n$-dimensional subvariety $X \subset Q_{2 n-2}$ whose Picard group is not generated by $H$.

The next two examples are chosen to see that the method can keep working even for nonhomogeneous ambient spaces.

Example 4.2. Let $Y$ be the blowing up of $\mathbb{P}^{6}$ at a point. Then the Picard group (and in fact the whole Chow ring) of $Y$ is generated by $H$ (the pullback of the hyperplane section of $\mathbb{P}^{6}$ ) and $E$ (the exceptional divisor). Let $X \subset Y$ be a smooth codimension-two subvariety and let $D$ be a smooth divisor on $X$. We write their classes in the Chow ring of $Y$ as:

From the intersection table in $X$ :

$$
\begin{aligned}
& {[X]=a_{1} H^{2}+a_{2} E^{2}} \\
& {[D]=\alpha_{1} H^{3}+\alpha_{2} E^{3}}
\end{aligned}
$$

\begin{tabular}{c|cccc} 
& $H^{3}$ & $E^{3}$ & $D H^{2}$ & $D E^{2}$ \\
\hline$H$ & $a_{1}$ & 0 & $\alpha_{1}$ & 0 \\
$E$ & 0 & $-a_{2}$ & 0 & $-\alpha_{2}$ \\
$D$ & $\alpha_{1}$ & $-\alpha_{2}$ & $\lambda_{1}$ & $\lambda_{2}$
\end{tabular}

we get that (1.4) takes the form:

$$
P:=\alpha_{1}^{2}-\alpha_{2}^{2}-a_{1} \lambda_{1}-a_{2} \lambda_{2}=0
$$

Then we see that we can get a decomposition

$$
P=-\frac{a_{1}-a_{2}}{a_{1}}\left|\begin{array}{cc}
a_{1} & \alpha_{1} \\
\alpha_{1} & \lambda_{1}
\end{array}\right|+\frac{1}{a_{1}}\left|\begin{array}{ccc}
a_{1} & 0 & \alpha_{1} \\
0 & -a_{2} & -\alpha_{2} \\
\alpha_{1} & -\alpha_{2} & \lambda_{1}+\lambda_{2}
\end{array}\right|
$$

The first determinant in this decomposition corresponds to the intersection matrix of the divisors $H$ and $D$ in the surface obtained as the intersection of $X$ with two general divisors of class $H$ (this is clearly a smooth irreducible surface and $H$ is very ample on it), hence by Hodge index theorem this determinant is nonpositive. Similarly, the second determinant corresponds to the intersection matrix of the divisors $H, E$ and $D$ in the surface obtained as the intersection of $X$ with two general divisors of class $H-E$. If we assume for instance that $H-E$ is ample on $X$, this surface is smooth irreducible, and hence the determinant is nonnegative by Hodge index theorem (observe that those three divisors generate the very ample divisor $2 H-E$ ). Since $a_{1}-a_{2}=(H-E)^{4}$ (hence strictly positive if we assume $H-E$ is ample) both determinants vanish and a simple argument shows that the rank of the intersection matrix (4.1) is two. As we have seen in the previous sections, this implies (under the assumption that $H-E$ is ample on $X$ ) that any divisor on $X$ depends numerically on $H$ and $E$. 
The interest of the next example is that shows that the method works even if the Picard group of the ambient variety is not a finitely generated group (and in particular it has torsion). It also shows (if $C=\mathbb{P}^{1}$ ) that the result of section 3 should remain valid for products of projective spaces of different dimension.

Example 4.3. Set $Y=C \times \mathbb{P}^{5}$, where $C$ is a smooth curve. The Picard group of $Y$ is generated by $H$ (the pull-back of the hyperplane section of $\mathbb{P}^{5}$ ) and the pull-back of the Picard group of $C$ by the projection $p_{1}: Y \rightarrow C$. From the numerical point of view, all the fibers of $p_{1}$ are equivalent, and we will denote with $F$ the class of a fiber. Let $X \subset Y$ be a smooth subvariety of codimension two and let $D \subset X$ be a smooth divisor. We can write the numerical classes of $X$ and $D$ as

$$
\begin{gathered}
{[X]_{\text {num }}=a_{1} H^{2}+a_{2} H F} \\
{[D]_{\text {num }}=\alpha_{1} H^{3}+\alpha_{2} H^{2} F}
\end{gathered}
$$

We consider now the intersection matrix:

\begin{tabular}{c|cccc} 
& $H_{\mid X}^{3}$ & $H_{\mid X}^{2} F_{\mid X}$ & $D H_{\mid X}^{2}$ & $D H_{\mid X} F_{\mid X}$ \\
\hline$H_{\mid X}$ & $a_{2}$ & $a_{1}$ & $\alpha_{2}$ & $\alpha_{1}$ \\
$F_{X}$ & $a_{1}$ & 0 & $\alpha_{1}$ & 0 \\
$D$ & $\alpha_{2}$ & $\alpha_{1}$ & $\lambda_{2}$ & $\lambda_{1}$
\end{tabular}

Equality (1.4) takes now the form:

$$
P:=2 \alpha_{1} \alpha_{2}-a_{1} \lambda_{2}-a_{2} \lambda_{1}
$$

which can be decomposed as

$$
P=\frac{1}{a_{1}}\left|\begin{array}{ccc}
a_{2} & a_{1} & \alpha_{2} \\
a_{1} & 0 & \alpha_{1} \\
\alpha_{2} & \alpha_{1} & \lambda_{2}
\end{array}\right|-\left|\begin{array}{cc}
a_{1} & \alpha_{1} \\
\alpha_{1} & \lambda_{1}
\end{array}\right|
$$

The first determinant in the decomposition corresponds to the intersection matrix of the classes of $H, F, D$ in the surface obtained by intersecting $X$ with two divisors of the class $H$, while the second determinant corresponds to the intersection matrix of the divisors $H, D$ in the surface obtained as the intersection of $X$ with a general fiber of $p_{1}$ and a hyperplane of $\mathbb{P}^{5}$. Under the appropriate assumptions for the irreducibility of those surfaces (even if $C=\mathbb{P}^{1}$, Debarre's result cannot provide the irreducibility of the intersection with $H F$ as in section 3), we can apply Hodge index theorem to conclude that both determinants are zero. In the usual way we conclude that the rank of the matrix in (4.2) has rank two and hence $D$ depends numerically on $H_{\mid X}$ and $F_{\mid X}$.

As these examples (and the rest of the paper) illustrates, our method works only numerically (and in a mysterious way: we guess that we are missing something deep that explains when the expression (1.4) can be decomposed as a sum of nonnegative summands). Only when there are some topological results (like the ones by Debarre) we are able to say something about the Picard group, but not as much as we would expect. In fact we suspect that, in the hypothesis of Theorems 2.1 and 3.1 one could conclude that the Picard group of $X$ is generated respectively by $H$ and $H_{1}, H_{2}$ (and more generally, that the cohomology coincides in the same range as Barth-Larsen theorems).

The fact that analogues of Barth-Larsen theorems seem to work in general suggests that also there should be a kind of Hartshorne's conjecture when $2 N<3 n$. However, the possible existence of low-rank vector bundles in general ambient spaces makes difficult to dare with a reasonable conjecture. For instance, $\mathbb{G}(1, n)$ has a universal rank-two vector bundle, so that any zero locus of a twist of it would be subcanonical of codimension two, but not a complete 
intersection. In this spirit, the first author suggested in [1, Conjecture 4.3] that the only smooth codimension-two subvarieties in $G(1, n)$ should be complete intersections or zero loci of twists of the rank-two universal quotient bundle. In 2 there is some evidence for this conjecture, proving it when $n=4$ and the degree of the variety is at most 25 .

In arbitrary codimension it seems very risky to state a similar conjecture for a general ambient space $Y$ of dimension $N$. One could expect that, under certain general conditions, the restriction map $H^{i}(Y, \mathbb{Z}) \rightarrow H^{i}(X, \mathbb{Z})$ is an isomorphism if $i \leq 2 n-N$. Hence, algebraic cycles on $X$ of codimension at most $\frac{2 n-N}{2}$ would lift to cycles on $Y$. Since the top Chern class of the normal bundle $N$ of $X$ in $Y$ always lifts to $X$ (by the self-intersection formula) this would mean that all the Chern classes of $N$ would lift to classes in $Y$ as long as $N-n-1 \leq \frac{2 n-N}{2}$. Thus a natural question is to ask under which conditions a smooth subvariety $X \subset Y$ is the zero locus of a vector bundle of rank $N-n$ on $Y$ if $3 N-2 \leq 4 n$ (the only non-trivial counterexample we know is the Segre embedding of $\mathbb{P}^{1} \times \mathbb{P}^{n-1}$ in $Q_{2 n-2}$ if $n$ is even). Observe that this inequality is stronger than the one in Hartshorne's conjecture except for $N=6$, in which our inequality includes the case $n=4$ (for which Hartshorne-Serre correspondence gives a positive answer).

Acknowledgements: We thank José Carlos Sierra and Giorgio Ottaviani for many useful discussions. It was José Carlos Sierra who pointed us the paper [6], and helped us to pass our results from numerical equivalence to linear equivalence.

\section{REFERENCES}

[1] E. Arrondo, Subcanonicity of codimension two subvarieties, Rev. Mat. Compl. 18 (2005), 69-80.

[2] E, Arrondo, M.L. Fania, Evidence to subcanonicity of codimension two subvarieties of $\mathbb{G}(1,4)$, to appear on Int. J. Math.

[3] W. Barth, Transplanting cohomology classes in complex projective space, Amer. J. Math. 92 (1970), 951-967.

[4] W. Barth, M.E. Larsen, On the homotopy-groups of complex projective manifolds, Math Scand. 30 (1972), 88-94.

[5] W. Barth and A. Van de Ven, On the geometry in codimension 2 of Grassmann manifolds, Lecture Notes in Math. 412, Springer Verlag (1974), 1-35.

[6] O. Debarre, Théorèmes de connexité pour les produits d'espaces projectifs et les grassmanniennes, Amer. J. Math. 118 (1996), no. 6, 1347-1367.

[7] R. Hartshorne, Varieties of small codimension in projective space, Bull. Amer. Math. Soc. 80 (1974), 1017-1032.

[8] S.L. Kleiman, D. Laksov, Schubert calculus, Amer. Math. Monthly 79 (1972), 1061-1082.

[9] M.E. Larsen, On the topology of projective manifolds, Invent. Math. 19 (1973), 251-260.

[10] A.J. Sommese, Complex subspaces of homogeneous complex manifolds. II. Homotopy results, Nagoya Math. J. 86, 101-129.

Departamento de Álgebra, Facultad de Ciencias Matemáticas, Universidad Complutense de MADRID, 28040 MADRID, SPAIN

E-mail address: arrondo@mat.ucm.es, jcaravan@mat.ucm.es 